TEMA Tend. Mat. Apl. Comput., 13, No. 1 (2012), 1-12.

doi: $10.5540 /$ tema.2012.013.01.0001

(C) Uma Publicação da Sociedade Brasileira de Matemática Aplicada e Computacional.

\title{
Um Modelo Matemático em Quimioterapia ${ }^{1}$
}

D.S. RODRIGUES ${ }^{2}$, Programa de Mestrado em Biometria, ${ }^{3}$ IBB, UNESP - Univ Estadual Paulista, Cx.P. 510, 18618-970 Botucatu, SP, Brasil.

S.T.R. PINHO 4 , Departamento de Física Geral, IF, UFBa, Campus Universitário de Ondina, 40210-340 Salvador, BA, Brasil.

P.F.A. MANCERA ${ }^{5}$, Departamento de Bioestatística, IBB, UNESP - Univ Estadual Paulista, Cx.P. 510, 18618-970 Botucatu, SP, Brasil.

Resumo. Modelo matemático aplicado em câncer é considerado para entender os motivos que levam a tratamentos quimioterápicos mal sucedidos. Frente às implicações do tratamento oncológico, as simulações numéricas possibilitaram a comparação de diferentes protocolos de tratamento, quanto a dose administrada e intervalo de tempo entre as doses. Conforme esperado clinicamente, nossos resultados indicam que a administração de baixas doses e longos intervalos de tempo entre as dosagens estão relacionados ao fracasso terapêutico.

Palavras-chave. Câncer, modelagem matemática, quimioterapia.

\section{Introdução}

Em relação a incidência de câncer no Brasil, o Instituto Nacional de Câncer estimou 489.270 novos casos da doença para o ano de 2010 (Brasil [5]). Quanto à ocorrência da doença em âmbito mundial, um relatório da Organização Mundial da Saúde aponta que a América do Norte tem a maior porcentagem de diagnósticos de cânceres em adultos, seguido pela Europa Ocidental, Austrália e Nova Zelândia (Pisani et al. [16]). Segundo Gatenby [9]: "Pacientes e políticos aguardam ansiosamente e reivindicam cada vez mais uma 'cura' para o câncer. Mas tentar controlar a doença pode revelar-se um plano melhor do que esforços para curá-la".

Dada a complexidade do câncer, a construção de modelos matemáticos da doença ainda permanece um grande desafio. Diversas metodologias têm sido empregadas, como equações diferenciais (Gatenby [8]), autômato celular (Reis et al. [17]), otimização (Panetta \& Fister [14]) e modelagem multi-escala (Stamatakos et al. [21]). Independentemente da metodologia adotada, certamente a associação entre teoria

\footnotetext{
${ }^{1}$ DSR: CAPES; PFAM: FAPESP 2010/20185-7, FUNDUNESP 00807/2010-DFP; STRP: FAPESB PNX 0006/2009, CNPq 305176/2009-9, INCT 57386/2008-9.

2 diegosarodrigues@gmail.com

${ }^{3}$ Endereço atual: SME-ICMC, USP, 13566-590, São Carlos.

${ }^{4}$ suani@ufba.br

${ }^{5}$ pmancera@ibb.unesp.br
}

Recebido em 16 Maio 2011; Aceito em 19 Outubro 2011. 
e experimentação provê melhores resultados, em que experimentos guiam teoria e teorias conduzem experimentos. Diante de tal junção, Skipper et al. [19] levantam a hipótese de que células tumorais são eliminadas em proporção constante a cada infusão de agente quimioterápico.

Uma das formas de tratamento do câncer é a quimioterapia antineoplásica, a qual envolve a administração de uma ou mais drogas para eliminar células tumorais. Almeja-se, nesta área, maximizar o efeito da droga nas células tumorais e minimizar efeitos colaterais no paciente, como por exemplo, náuseas e vômitos, experimentados por aproximadamente $25 \%$ dos pacientes. Apesar do sucesso da quimioterapia e dos avanços em biologia do câncer, ainda permanecem em aberto questões cruciais como, por exemplo, se a quimioterapia deve preceder a cirurgia ou vice-versa, e a determinação de protocolos ótimos de tratamento. Neste cenário, o primeiro passo é entender em quais situações a quimioterapia fracassa. Para tanto, este trabalho trata de estudos matemáticos sobre duas dessas ocasiões nas quais esta forma de terapia é mal sucedida.

Este artigo é estruturado como se segue: apresentação e justificativa do modelo matemático, seguido de resultados analíticos e numéricos para os casos tratado e não tratado da doença e finalmente conclusões.

\section{Modelo}

A poliquimioterapia é largamente empregada nos dias de hoje e tem gerado avanços no tratamento de câncer, porém, quantitativamente, pouco se conhece sobre o efeito combinado de drogas. Por exemplo, um agente quimioterápico pode potencializar o efeito de outro ${ }^{6}$, o que implica em respostas terapêuticas nem sempre aditivas. Assim sendo, compreendemos aqui tratamentos nos quais há administração de apenas um dado agente quimioterápico. Especialmente, escolhemos as drogas ciclo-inespecíficas, pois estas são mais dose-dependentes do que as ciclo-específicas e, além disto, porque atuam igualmente sobre as células proliferativas e não proliferativas do tumor, as quais são indistintas perante o modelo apresentado. Dentre as modalidades de quimioterapia, abordamos somente a neoadjuvante, pois neste tratamento utiliza-se somente drogas.

Se considerarmos uma situação na qual inibição e estímulos angiogênicos estão em equilíbrio antes do tratamento se iniciar e, a seguir, que o tratamento se inicia, porém não apresenta efeito antiangiongênico e denotando-se o número de células tumorais e normais por $N_{i}(i=1,2)$ e a quantidade do agente quimioterápico por $Q$, propomos o seguinte modelo:

$$
\left\{\begin{aligned}
\frac{d N_{1}}{d t} & =r_{1} N_{1}\left(1-\frac{N_{1}}{k_{1}}-\frac{\alpha_{1} N_{2}}{k_{1}}\right)-\frac{\mu N_{1} Q}{a+Q} \\
\frac{d N_{2}}{d t} & =r_{2} N_{2}\left(1-\frac{N_{2}}{k_{2}}-\frac{\alpha_{2} N_{1}}{k_{2}}\right)-\frac{\nu N_{2} Q}{b+Q} \\
\frac{d Q}{d t} & =q(t)-\lambda Q
\end{aligned}\right.
$$

\footnotetext{
${ }^{6}$ Por exemplo, segundo Bonassa [3], o emprego individual de ciclofosfamida ou doxorrubicina, ocasiona $30 \%$ de resposta favorável em pacientes portadoras de câncer de mama avançado, enquanto a administração conjunta provoca 70 a $80 \%$.
} 
em que o índice $i=1$ diz respeito à população de células tumorais e $i=2$ às normais, $r_{i}$ denota a taxa de crescimento das populações tumoral e normal, $k_{1}$ denota a capacidade suporte do tumor após a neovascularização atingir o equilíbrio, $k_{2}$ é a capacidade suporte das células normais, determinada pelo tamanho do órgão, $\alpha_{i}$ o coeficiente de competição interespecífica referente à população $i, a$ e $b$ determinam a saturação do efeito resposta a droga, $\lambda$ é a taxa de decaimento de um dado agente quimioterápico ciclo-inespecífico, $\mu$ é a taxa de tratamento das células tumorais e $\nu$ é taxa de mortalidade das células normais em decorrência do tratamento, já que a quimioterapia também afeta as células normais.

A interação entre as células normais e tumorais é dada por Lotka-Volterra [8], pois Vaidya \& Alexandro-Jr [22] relatam circunstância na qual o modelo logístico é o mais adequado para o ajuste de dados de crescimento para humanos. Para via de administração intravenosa do medicamento, a variação temporal da quantidade de droga é dada pela cinética de primeira ordem (Lüllmann et al. [10]), como dado pela última equação de (2.1). Sobre o efeito da droga, consideramos resposta funcional do tipo Michaelis-Menten, pois após uma certa dose, a resposta ao tratamento independe da quantidade de droga, sendo a taxa de mortalidade per capita das células tumorais dada por $\mu Q /(a+Q)$. No contexto de modelagem em câncer, Aroesty et al. [1] mostraram que tal resposta funcional permite descrever dinâmica tumoral sobre quimioterapia ciclo-inespecífica. Admitimos ainda que as células são eliminadas pela droga em proporção constante, como postula (Skipper et al. [19]).

Para que o tratamento faça sentido, o agente quimioterápico tem que agir com maior intensidade nas células tumorais. Por exemplo, segundo Buick [6], o efeito de droga em linfomas é até $10^{4}$ vezes maior do que em células de médula óssea, donde temos que

$$
\mu \gg \nu \text {. }
$$

Quanto às taxas de crescimento, temos que o tumor cresce mais rapidamente do que os tecidos normais pelo fato das células tumorais conseguirem adiar a morte celular programada, chamada apoptose. Se $r_{i}$ é dado por $r_{i}=b_{i}-d_{i}$, em que $b_{i}$ é a taxa de divisão celular e $d_{i}$ é a taxa de morte celular, então do ponto de vista biológico $b_{1} \approx b_{2}$, mas devido à evasão da apoptose das células tumorais, $d_{1}<d_{2}$, implicando em

$$
r_{1}>r_{2} .
$$

Sobre o tratamento, há situações nas quais o medicamento é infundido ininterruptamente por alguns dias (Baxter [2]). Se tal administração se dá a uma taxa constante, então $q(t)=q>0$, que corresponde a situação de decaimento exponencial do agente quimioterápico. Entretanto, na maioria dos protocolos, administra-se droga(s) em intervalos de tempo fixos e assim $q(t)$ é uma função periódica. Neste último caso, como o tempo gasto na infusão é muito menor que o tempo total de tratamento do paciente, admitimos que a droga interage imediatamente com o tumor. Denominamos estas duas situações como:

1. Administração contínua: $q(t)=q$ (constante) $>0$.

2. Administração em ciclos: Nesta forma de administração, cada ciclo ${ }^{7}$ dura

\footnotetext{
${ }^{7}$ Ciclo é definido como período após o qual o protocolo de tratamento se repete.
} 
normalmente três ou quatro semanas, seguido por um período de descanso, no qual o quimioterápico não é administrado ${ }^{8}$. De acordo com Martin \& Teo [11], definimos a infusão da seguinte forma:

$$
q(t)=\left\{\begin{array}{l}
q>0, \quad n \leq t<n+\tau \\
0, \quad n+\tau \leq t<n+T
\end{array}\right.
$$

em que $T$ é o ciclo (intervalo de tempo do ciclo), $n=0, T, 2 T, \ldots$, e $\tau$ é tempo de infusão, de modo que $T>>$.

Quanto às informações quantitativas desta forma de administração, há um esquema de tratamento para câncer de mama estabelecido pelo Ministério da Saúde [4], o protocolo FAC (fluorouracil, doxorrubicina e ciclofosfamida), que pressupõe a aplicação de ciclofosfamida IV (intravenosa) em bolus ${ }^{9}$ na dose de $500 \mathrm{mg}$ por metro quadrado de superfície corporal do paciente, a cada 21 dias. Adotamos tal protocolo, considerando que o tratamento se dá apenas com ciclofosfamida. A partir da fórmula de Mosteller [12], estimamos que a superfície corporal de um paciente de massa $70 \mathrm{~kg}$ e de estatura $1,70 \mathrm{~m}$ é $1,8 \mathrm{~m}^{2}$, estabelecendo assim dose de $900 \mathrm{mg}$ por ciclo, infundida em três horas. Denominaremos este esquema de protocolo padrão, e, a partir de (2.4) vamos definí-lo como $q=900 \mathrm{mg} /(1 / 8 \mathrm{dia})=7200 \mathrm{mg} / \mathrm{dia}$, $\tau=1 / 8$ dia, $T=21$ dias, $n=0,21,42,63$ (quatro infusões) e $q(t \geq 84) \equiv 0$.

A respeito das situações nas quais ocorre o fracasso clínico da quimioterapia, Norton [13] aponta cinco causas: 1) Tumor resistente ao tratamento devido à terapia ser iniciada para tumor de grande volume com baixa fração de crescimento; 2) Tumor não curado devido à administração de doses de intensidades insuficientes para manter a regressão do tumor (devido a regressão relativamente baixa de pequenos tumores); 3) Surgimento de segundo tumor resistente por mutação ou seleção; 4) Tumor resistente ao tratamento devido à administração de dose muito baixa; 5) Tumor resistente ao tratamento porque o intervalo de tempo entre as doses é muito alto. Dentre as razões listadas anteriormente, 1), 2) e 3) não são abordadas aqui, já que, no modelo que propomos, não há distinção entre as células tumorais. Os itens 4) e 5) são contemplados aqui, para administração em ciclos. Para tal investigação, a fim de impor condições sobre a cura espontânea do câncer e de modo similar a Freedman \& Nani [7] e Pinho et al. [15], postulamos que a eliminação do tumor jamais ocorre na ausência de tratamento, constituindo o que chamaremos hipótese geral do câncer.

O modelo para o caso sem tratamento da doença é dado por Gatenby [8]

$$
\left\{\begin{array}{l}
\frac{d N_{1}}{d t}=r_{1} N_{1}\left(1-\frac{N_{1}}{k_{1}}-\frac{\alpha_{1} N_{2}}{k_{1}}\right), \\
\frac{d N_{2}}{d t}=r_{2} N_{2}\left(1-\frac{N_{2}}{k_{2}}-\frac{\alpha_{2} N_{1}}{k_{2}}\right) .
\end{array}\right.
$$

Gatenby [8] apresentam análise de estabilidade local do sistema (2.5). No entanto, procedemos com os resultados a seguir para estabelecer a hipótese geral do câncer e comparar os resultados numéricos e constatações biológicas.

\footnotetext{
${ }^{8}$ Protocolos de tratamento são estabelecidos dessa forma, para que o paciente possa se recuperar a cada sessão de quimioterapia.

${ }^{9} \mathrm{Na}$ técnica de administração bolus a droga é injetada de uma vez, via intravenosa.
} 


\section{Resultados e Discussões}

\subsection{Câncer não tratado}

As soluções de equilíbrio do sistema (2.5) são $F_{1}(0,0)$ (extinção das células normais e tumorais); $F_{2}\left(0, k_{2}\right)$ (cura espontânea); $F_{3}\left(k_{1}, 0\right)$ (extinção de células normais e persistência do tumor); $F_{4}\left(\frac{\alpha_{1} k_{2}-k_{1}}{\alpha_{2} \alpha_{1}-1}, \frac{\alpha_{2} k_{1}-k_{2}}{\alpha_{2} \alpha_{1}-1}\right)$ (coexistência entre as células normais e tumorais).

Biologicamente, temos que $F_{1}(0,0)$ e $F_{2}\left(0, k_{2}\right)$ nunca são observadas, sendo portanto instáveis segundo a hipótese geral do câncer. Vejamos sob quais condições isto ocorre.

Os autovalores da matriz jacobiana avaliada em $F_{1}(0,0)$ são $r_{1}>0$ e $r_{2}>0$, e então $F_{1}(0,0)$ é um nó hiperbólico instável, e como $N_{1}(0) \neq 0$ e $N_{2}(0) \neq 0$, então na ausência de tratamento da doença as células normais e tumorais nunca são extintas simultaneamente.

Os autovalores associados a matriz jacobiana calculada em $F_{2}\left(0, k_{2}\right)$ são $-r_{2}$ e $\left(r_{1}\left(k_{1}-\alpha_{1} k_{2}\right)\right) / k_{1}$. Como $-r_{2}<0$, a condição de instabilidade de $F_{2}\left(0, k_{2}\right)$ é

$$
k_{1}-\alpha_{1} k_{2}>0 \quad \Longrightarrow \quad 0<\alpha_{1}<\frac{k_{1}}{k_{2}} \text {. }
$$

Assim, impondo a desigualdade (3.1), decorre que a solução de cura espontânea $F_{2}\left(0, k_{2}\right)$ é ponto de sela hiperbólico e a hipótese geral do câncer é garantida. Mesmo que a desigualdade (3.1) seja satisfeita, o ponto de equilíbrio de coexistência $F_{4}\left(\frac{\alpha_{1} k_{2}-k_{1}}{\alpha_{2} \alpha_{1}-1}, \frac{\alpha_{2} k_{1}-k_{2}}{\alpha_{2} \alpha_{1}-1}\right)$ possui valores não negativos para $N_{1}$ e $N_{2}$, se $\alpha_{2} \alpha_{1}-1<0$ e $\alpha_{2} k_{1}-k_{2}<0$. Assim, sob a hipótese geral do câncer, apresentamos na Figura 1 uma simulação numérica na qual as células normais e tumorais coexistem.

\subsection{Tratamento quimioterápico anti-neoplásico}

Nesta subseção, primeiro exibiremos a análise e resultados para a administração contínua, $q>0$, e, em seguida, para a administração em ciclos.

\section{Administração contínua}

Quando $q(t)=q>0$, o sistema (2.1) torna-se autônomo e possui quatro pontos de equilíbrio, dados por $G_{1}\left(0,0, \frac{q}{\lambda}\right)$ (eliminação das células normais e tumorais devido a alta dosagem administrada); $G_{2}\left(0, \breve{N}_{2}, \frac{q}{\lambda}\right)$ (cura da doença através do tratamento quimioterápico neoadjuvante); $G_{3}\left(\breve{N}_{1}, 0, \frac{q}{\lambda}\right)$ (eliminação das células normais pelo tratamento com persistência do tumor); $G_{4}\left(\overline{N_{1}}, \overline{N_{2}}, \frac{q}{\lambda}\right)$ (coexistência entre células normais e tumorais na presença do tratamento), em que

$$
\breve{N}_{1}=\frac{k_{1}\left(r_{1} a \lambda+q\left(r_{1}-\mu\right)\right)}{r_{1}(a \lambda+q)}, \quad \breve{N}_{2}=\frac{k_{2}\left(r_{2} b \lambda+q\left(r_{2}-\nu\right)\right)}{r_{2}(b \lambda+q)} .
$$

Os pontos $G_{1}\left(0,0, \frac{q}{\lambda}\right)$ e $G_{3}\left(\breve{N}_{1}, 0, \frac{q}{\lambda}\right)$ representam situações que de fato não são observadas no estado de equilíbrio: $G_{1}$ representa a eliminação do tumor e também do orgão no qual ele se aloja; $G_{3}$ diz respeito a uma situação na qual a terapia 


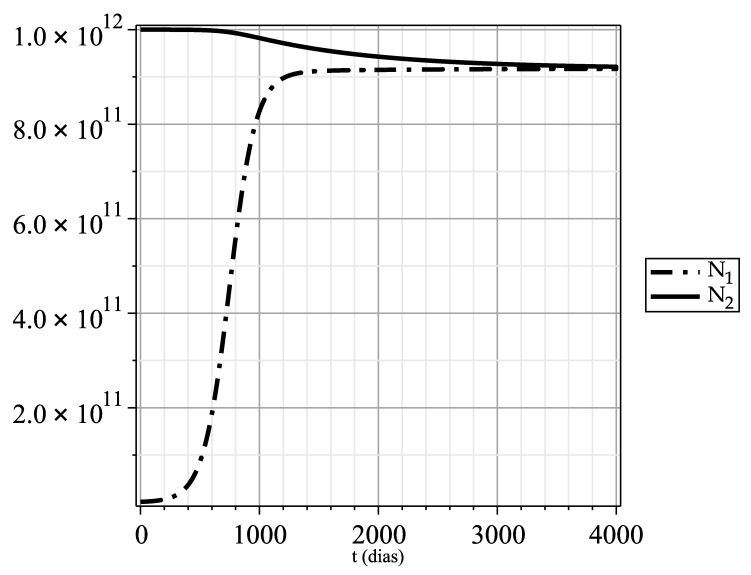

Figura 1: Evolução do tumor quando o paciente (humano) não é submetido ao tratamento (sistema $(2.5)$ ). Condições iniciais: $N_{1}(0)=10^{9}$ células tumorais $(\approx$ $1 \mathrm{~cm}$ de diâmetro, clinicamente palpável) e $N_{2}(0)=10^{12}$ células normais, em que os valores de $r_{1}, r_{2}, k_{1}, k_{2}, \alpha_{1}$ e $\alpha_{2}$ são dados na Tabela 1 . Embora, a simulação seja exibida até 4000 dias, dificilmente o paciente sobreviveria após, aproximadamente, 1500 dias, devido às complicações decorrentes de um tumor de $9 \times 10^{11}$ células $(\approx 900 \mathrm{~g})$.

elimina o orgão hospedeiro, porém sem eliminação do tumor. Ainda assim, a análise de estabilidade de tais pontos é importante, pois nos fornece as condições sob as quais $G_{1}$ e $G_{3}$ são instáveis, assim como fora explicado acima.

Já o ponto $G_{4}\left(\overline{N_{1}}, \overline{N_{2}}, \frac{q}{\lambda}\right)$ não representa a cura, mas ainda assim pode indicar situações de terapia bem ou mal sucedida clinicamente, dependendo da razão entre $\overline{N_{1}}$ e $N_{1}(0)$. Se $\overline{N_{1}} \ll N_{1}(0)$ e se $N_{1}(0)$ representar um tumor relativamente grande $\left(\geq 10^{10}\right.$ células, $\approx 100 \mathrm{~g}$ ), então $G_{4}$ indica o sucesso clínico do tratamento, pois neste caso a redução tumoral é acentuada.

Para que $G_{2}\left(0, \breve{N}_{2}, \frac{q}{\lambda}\right)$ tenha sentido do ponto de vista biológico, temos

$$
\breve{N}_{2}>0 \Longrightarrow 0<q<\frac{r_{2} b \lambda}{\nu-r_{2}}, \quad \text { com } \quad \nu>r_{2}
$$

A desigualdade (3.3) estabelece um limite superior para a taxa de infusão de droga necessária para a cura. Analogamente, a condição necessária e suficiente para que $\breve{N}_{1}$ seja positivo em $G_{3}\left(\breve{N}_{1}, 0, \frac{q}{\lambda}\right)$ é

$$
0<q<\frac{r_{1} a \lambda}{\mu-r_{1}}, \quad \text { com } \mu>r_{1} .
$$

Avaliada em $G_{1}\left(0,0, \frac{q}{\lambda}\right)$, a matriz jacobiana tem os seguintes autovalores

$$
\Theta_{1}=\frac{r_{1} a \lambda+q\left(r_{1}-\mu\right)}{a \lambda+q}, \quad \Theta_{2}=\frac{r_{2} b \lambda+q\left(r_{2}-\nu\right)}{b \lambda+q}, \quad \Theta_{3}=-\lambda .
$$


O ponto de equilíbrio $G_{1}\left(0,0, \frac{q}{\lambda}\right)$ tem que ser instável; caso contrário o tratamento quimioterápico eliminaria as células tumorais e também as células normais. Como $\lambda>0$, então $\Theta_{3}<0$. Portanto, basta que $\Theta_{1}$ ou $\Theta_{2}$ seja positivo para que $G_{1}\left(0,0, \frac{q}{\lambda}\right)$ seja instável. Assim, se $\Theta_{1}>0, G_{1}\left(0,0, \frac{q}{\lambda}\right)$ é instável e $\breve{N}_{1}>0$ em $G_{3}\left(\breve{N}_{1}, 0, \frac{q}{\lambda}\right)$. Analogamente, se $\Theta_{2}>0, G_{1}\left(0,0, \frac{q}{\lambda}\right)$ é instável e $\breve{N}_{2}>0$ em $G_{2}\left(0, \breve{N}_{2}, \frac{q}{\lambda}\right)$.

A seguir, apresentamos sob quais condições o modelo prevê eliminação de tumor pelo tratamento quimioterápico. A matriz jacobiana avaliada em $G_{2}\left(0, \breve{N}_{2}, \frac{q}{\lambda}\right)$ é dada por

$$
\mathbb{N}\left(0, \breve{N}_{2}, \frac{q}{\lambda}\right)=\left(\begin{array}{ccc}
n_{11} & 0 & 0 \\
-\frac{r_{2} \breve{N}_{2} \alpha_{2}}{k_{2}} & n_{22} & \frac{-\nu \breve{N}_{2} \lambda^{2} b}{(b \lambda+q)^{2}} \\
0 & 0 & -\lambda
\end{array}\right)
$$

em que $n_{11}=\frac{r_{1}(a \lambda+q)\left(k_{1}-\alpha_{1} \breve{N}_{2}\right)-\mu q k_{1}}{k_{1}(a \lambda+q)} \quad$ e $\quad n_{22}=\frac{r_{2}(b \lambda+q)\left(k_{2}-2 \breve{N}_{2}\right)-\nu q k_{2}}{k_{2}(b \lambda+q)}$. O polinômio característico $P(\psi)$ de $\mathbb{N}\left(0, \breve{N}_{2}, \frac{q}{\lambda}\right)$ é dado por

$$
P(\psi)=\frac{(\lambda+\psi) R(\psi) D(\psi)}{k_{1} k_{2}(a \lambda+q)(b \lambda+q)},
$$

em que

$$
\begin{aligned}
& \left.R(\psi)=(b \lambda+q)\left(k_{2}\left(r_{2}-\psi\right)-2 r_{2} \breve{N}_{2}\right)\right)-\nu q k_{2}, \\
& \left.D(\psi)=(a \lambda+q)\left(k_{1}\left(r_{1}-\psi\right)-r_{1} \alpha_{1} \breve{N}_{2}\right)\right)-\mu q k_{1} .
\end{aligned}
$$

Então, $\psi_{1}=-\lambda<0$ é uma raiz de $P(\psi)$. Os outros autovalores restantes são as duas raízes dos polinômios $R(\psi)$ e $D(\psi)$. Substituindo $\breve{N}_{2}=\frac{k_{2}\left(r_{2} b \lambda+q\left(r_{2}-\nu\right)\right)}{r_{2}(b \lambda+q)} \mathrm{em}$ (3.8), obtemos que $\psi_{2}=-\Theta_{2}$ é raiz de $R(\psi)$. Deste modo, se a desigualdade $\nu>r_{2}$ for verificada, então $\psi_{2}<0$. O terceiro autovalor $\psi_{3}$ é encontrado substituindo-se (3.2) em (3.9), a saber

$$
\psi_{3}=\frac{\mathcal{A} q^{2}+\mathcal{B} q+\mathcal{C}}{k_{1} r_{2}(a \lambda+q)(b \lambda+q)}
$$

em que

$$
\begin{aligned}
\mathcal{A} & =r_{2} k_{1}\left(r_{1}-\mu\right)+\alpha_{1} k_{2} r_{1}\left(\nu-r_{2}\right) \\
\mathcal{B} & =(a+b)\left(r_{1} r_{2} \lambda\left(k_{1}-\alpha_{1} k_{2}\right)\right)+\lambda\left(r_{1} a \nu \alpha_{1} k_{2}-r_{2} b \mu k_{1}\right), \\
\mathcal{C} & =r_{1} r_{2} a b \lambda^{2}\left(k_{1}-\alpha_{1} k_{2}\right) .
\end{aligned}
$$

Para que o ponto de equilíbrio $G_{2}\left(0, \breve{N}_{2}, \frac{q}{\lambda}\right)$ seja localmente assintoticamente estável, além da condição $\nu>r_{2}, \psi_{3}$ tem que negativo. Assim, utilizando (3.10), desejamos que

$$
\frac{\mathcal{A} q^{2}+\mathcal{B} q+\mathcal{C}}{k_{1} r_{2}(a \lambda+q)(b \lambda+q)}<0 \quad \Longrightarrow \quad \mathcal{A} q^{2}+\mathcal{B} q+\mathcal{C}<0 .
$$


Substituindo (3.11)-(3.13) em (3.14), temos que $\mathcal{D} \alpha_{1}-\mathcal{E}<0$, em que

$$
\begin{aligned}
\mathcal{D} & =-r_{1} k_{2}(a \lambda+q)\left(r_{2}(b \lambda+q)-\nu q\right), \\
\mathcal{E} & =-r_{2} k_{1}(b \lambda+q)\left(r_{1}(a \lambda+q)-\mu q\right),
\end{aligned}
$$

resultando em

$$
\mathcal{D} \alpha_{1}<\mathcal{E}
$$

De (3.3) e (3.4), decorre respectivamente que $\mathcal{D}<0$ e $\mathcal{E}<0$. Como $\mathcal{D}<0$, então

$$
\alpha_{1}>\frac{\mathcal{E}}{\mathcal{D}}
$$

Assim, $\frac{\mathcal{E}}{\mathcal{D}}>0$ e para $\frac{\mathcal{E}}{\mathcal{D}}<\frac{k_{1}}{k_{2}}, G_{2}\left(0, \breve{N}_{2}, \frac{q}{\lambda}\right)$ é localmente assintoticamente estável se as desigualdades $\nu>r_{2},(3.1)$ e (3.18) forem satisfeitas. Tais desigualdades combinadas resultam em

$$
\frac{\mathcal{E}}{\mathcal{D}}<\alpha_{1}<\frac{k_{1}}{k_{2}}
$$

Por fim, se $\frac{\mathcal{E}}{\mathcal{D}} \geq \frac{k_{1}}{k_{2}}$, então sob a hipótese geral do câncer $G_{2}\left(0, \breve{N}_{2}, \frac{q}{\lambda}\right)$ é instável.

Em suma, derivamos os seguintes resultados para administração contínua (e constante):

- Estabelecemos valores limiares para a infusão da droga, aquém dos quais a quimioterapia não elimina simultaneamente o tumor e o orgão hospedeiro isto ocorre quando $G_{1}\left(0,0, \frac{q}{\lambda}\right)$ é instável, ou seja, quando a desigualdade (3.3) ou a desigualdade (3.4) é satisfeita;

- Apresentamos as condições sob as quais a cura da doença é atingida, ou seja, quando $G_{2}\left(0, \breve{N}_{2}, \frac{q}{\lambda}\right)$ é estável, ou seja, $\nu>r_{2}$ e quando a desigualdade (3.19) é satisfeita;

- As condições para $G_{3}\left(\breve{N}_{1}, 0, \frac{q}{\lambda}\right)$ sob as quais a quimioterapia elimina as células normais, mas não elimina o tumor são obtidas fazendo-se a mesma análise ora apresentada para $G_{2}\left(0, \breve{N}_{2}, \frac{q}{\lambda}\right)$, devido a simetria do sistema de equações.

A seguir, descrevemos, através do modelo apresentado, a hipótese log-kill (Skipper et. al. [19]), hipótese esta caracterizada pela eliminação das células tumorais em proporção constante, a cada infusão de agente quimioterápico (morte celular logaritmica). Por exemplo, para uma massa tumoral de $10^{10}$ células, uma redução de três logs elimina $99,9 \%$ do tumor, pois $10^{7} / 10^{10}=0,001=0,1 \%$. Além de abordar esta hipótese, apresentamos também resultados que concordam com Norton [13] no que diz respeito ao fracasso de protocolos de quimioterapia antineoplásica.

\section{Administração em ciclos}

Apresentamos a seguir simulações numéricas para o modelo com administração em ciclos. Utilizando o protocolo padrão, investigamos o fracasso do tratamento 
Tabela 1: Parâmetros das simulações numéricas para câncer humano.

\begin{tabular}{|c|c|c|c|}
\hline Parâmetro & Valor & Unidade & Referência/comentário \\
\hline$r_{1}$ & $10^{-2}$ & $\mathrm{dia}^{-1}$ & Spratt et al. [20] \\
\hline$r_{2}$ & $10^{-3}$ & $\mathrm{dia}^{-1}$ & $r_{2}<r_{1}(\operatorname{ver}(2.3))$ \\
\hline$k_{1}$ & $10^{12}$ & células & Spratt et al. [20] e Weinberg [23] \\
\hline$k_{2}$ & $10^{12}$ & células & $k_{2} \sim k_{1}$ \\
\hline$\alpha_{1}$ & $9 \times 10^{-2}$ & - & $\alpha_{1}<\frac{k_{1}}{k_{2}}$ (hipótese geral do câncer) \\
\hline$\alpha_{2}$ & $9 \times 10^{-2}$ & - & valor assumido $^{\S}$ \\
\hline$\mu$ & 8 & $\mathrm{dia}^{-1}$ & valor assumido $^{\S}$ \\
\hline$\nu$ & $8 \times 10^{-2}$ & $\mathrm{dia}^{-1}$ & $\nu \ll \mu(\operatorname{ver}(2.2))$ \\
\hline$\lambda$ & 4,16 & $\mathrm{dia}^{-1}$ & Baxter $[2] \dagger$ \\
\hline$a$ & $2 \times 10^{3}$ & $\mathrm{mg}$ & valor assumido ${ }^{\S}$ \\
\hline$b$ & $5 \times 10^{6}$ & $\mathrm{mg}$ & valor assumido ${ }^{\S}$ \\
\hline
\end{tabular}

†O valor de $\lambda$ foi calculado a partir da meia-vida de quatro horas da ciclofosfamida (Baxter [2]). $\S N a ̃ o$ encontramos referências de medidas experimentais para tais parâmetros. Assim sendo, adotamos valores segundo a ordem de grandeza das interações célula-célula e célula-droga.

devido à administração de dose muito baixa, bem como, quando o intervalo de tempo entre as doses é muito alto. Nas simulações numéricas exibidas na Figura 2 utilizamos os parâmetros listados na Tabela 1. Em particular, nas Figuras 2(a)-(b), observamos que o modelo proposto para tratamento em ciclos apresenta eliminação de células tumorais em proporção constante, assim como postula a hipótese log-kill. Estes resultados quantitativos para ciclofosfamida são similares ao de Stamatakos et al. [21] para a epirrubicina, diferindo-se apenas pela ausência de flutuações no número de células tumorais, que não foram encontradas, pois, ao contrário de Stamatakos et al. [21], além do nosso modelo ser determinístico, é também contínuo.

Na Figura 2(a), comparamos o protocolo padrão com outro protocolo cuja dosagem é mais baixa, na qual nota-se uma redução tumoral bem mais acentuada no protocolo padrão, ao passo que no outro protocolo não há alteração da ordem de grandeza do tumor (o mesmo mantém-se da ordem de $\approx 10^{10}$ células), explicando assim porque baixas doses implicam no fracasso terapêutico. Na Figura 2(b), exibimos simulação de dois ciclos utilizados na prática clínica: infusão a cada três semanas - protocolo padrão e de outro protocolo cujo ciclo é maior (quatro semanas), sendo que não há diferença considerável entre os tamanhos mínimos do tumor em cada protocolo.

Podemos afirmar, portanto, que não há diferença clínica para infusão a cada três ou quatro semanas. Entretanto, num esquema de terapia dado pelo protocolo padrão, porém com ciclo relativamente grande (aproximadamente 50 dias ou mais) ocorre o fracasso do tratamento, já que após a administração da droga, o tumor ressurge e atinge o tamanho que tivera antes da infusão. Nesse protocolo hipotético, seriam eliminadas apenas poucas células tumorais que surgiriam no período, não surtindo efeito terapêutico. 
(a)

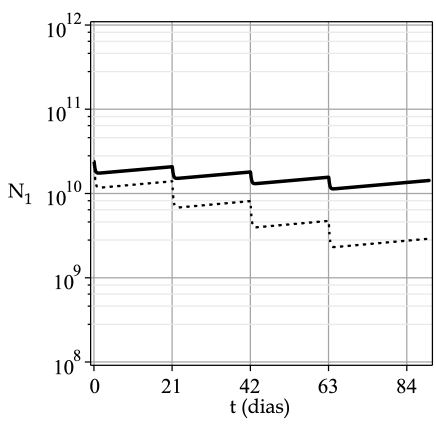

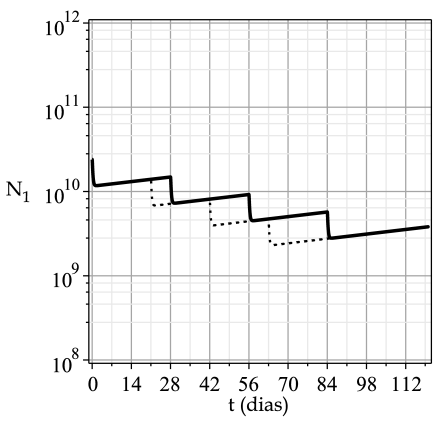

Figura 2: Tratamento quimioterápico neoadjuvante. Condições iniciais: $N_{1}(0)=$ $2,4 \times 10^{10}$ células tumorais, $N_{2}(0)=10^{12}$ células normais e $Q(0)=0$, e valores dos parâmetros contidos na Tabela 1. (a) Tratamento quimioterápico mal sucedido devido a administração de dose muito baixa (linha contínua), em que a curva com linha tracejada representa o bem sucedido protocolo padrão, pois neste ocorre significatica redução tumoral de $10^{10}$ células para $10^{9}$ células (isto indica que decorridos três meses após o início do tratamento o tumor reduz-se para $\approx 1 g$ ). O ciclo $T$ é de 21 dias e o tempo $\tau$ de infusão 3 horas (1/8 dia). Dose (linha contínua): $370 \mathrm{mg}$ de ciclofosfamida; infusão: $q=8 \times 370 \mathrm{mg} /$ dia. (b) Comparação entre tratamento quimioterápico neoadjuvante para ciclo de 28 dias (linha contínua) e protocolo padrão, no qual o ciclo dura 21 dias; praticamente não há diferença de tamanho do tumor: do ponto de vista clínico tais protocolos resultam na mesma resposta do paciente à terapia.

\section{Conclusões}

O modelo de equações diferenciais ordinárias que propomos captura a característica log-kill dos tratamentos nos quais há administração de apenas um dado agente quimioterápico ciclo-inespecífico. Utilizando dados experimentais para alguns parâmetros, verificamos que o efeito log-kill ocorre também para o modelo logístico. Para a administração contínua da droga, todas as condições de estabilidade da solução de cura dependem da taxa de infusão da droga. Assim sendo, o fluxo de infusão de droga determina a cura do tumor ou apenas a sua redução parcial.

Para administração em ciclos, obtivemos resultado qualitativo para ciclofosfamida análoga a simulação dada pelo modelo discreto multiescala de Stamatakos et al. [21] para o tratamento quimioterápico neoadjuvante do câncer de mama com epirrubicina, mas para um modelo contínuo e mais simples.

O modelo de tratamento quimioterápico neoadjuvante proposto permite comparar diferentes protocolos, quanto à dose administrada e também suscita a discussão sobre a redução do intervalo de tempo entre as dosagens, questão esta que será investigada especialmente em nosso próximo trabalho [18]. Assim como descrito por Norton [13], as simulações numéricas apresentadas apontam que a administração de baixa dose ou longo intervalo de tempo entre as infusões do agente quimioterápico resulta em tratamentos falhos. Assim sendo, frente as estratégias de ad- 
ministração da quimioterapia antineoplásica, o modelo proposto provê explicação analítico-quantitativa para fracassos clínicos de protocolos de tratamento de câncer.

\section{Referências}

[1] J. Aroesty, T. Lincoln, N. Shapiro, G. Boccia, Tumor growth and chemotherapy: mathematical methods, computer simulations, and experimental foundations, Math. Biosc., 17 (1973), 243-300.

[2] Baxter, Genuxal (ciclofosfamida), em "Guia Prático de Prescrição e Consulta", (Alamtec, ed.), São Paulo, Aquaprint, 2005.

[3] E.M.A. Bonassa, "Enfermagem em quimioterapia", São Paulo, Livraria Atheneu Editora, 1992.

[4] Brasil, Bases do tratamento, em "Ações de Enfermagem para o Controle do Câncer: uma proposta de integração de ensino", (INCA, ed.), Rio de Janeiro, INCA, 2008.

[5] Brasil, "Estimativas 2010: incidência de câncer no Brasil", Rio de Janeiro, INCA, 2010. 212p.

[6] R.N. Buick, Cellular basis of chemotherapy, em "Cancer Chemotherapy Handbook", (R.T. Dorr, D.D.V. Hoff, eds.), Appleton and Lange, 1994. p.9.

[7] H.I. Freedman, F.K. Nani, "A Mathematical Model of Cancer Treatment by Chemotherapy", Preprint, 1-38, 1998.

[8] R.A. Gatenby, Application of competition theory to tumour growth: implications for tumour biology and treatment, Eur. J. Cancer, 32A (1996), 722-726.

[9] R.A. Gatenby, A change of strategy in the war on cancer, Nature, 459 (2009), 508-509.

[10] H. Lüllmann, K. Mohr, A. Ziegler, D. Bieger, "Color Atlas of Pharmacology", New York, Thieme Stuttgart, 2000.

[11] R. Martin, K.L. Teo, "Optimal Control of Drug Administration in Cancer Chemotherapy", Singapore, World Scientific, 1993.

[12] R.D. Mosteller, Simplified calculation of body surface area, N. Engl. J. Med., 1098, 1987.

[13] L. Norton, Introduction, em "A Synopsis of Cancer Chemotherapy", (R.T. Silver, R.D. Lauper, C.I. Jarowski, eds.), New York, York Medical Books, 1987. p.24.

[14] J.C. Panetta, K.R. Fister, Optimal control applied to competing chemotherapeutic cell-kill strategies, SIAM J. Appl. Math., 63 (2003), 1954-1971. 
[15] S.T.R. Pinho, H.I. Freedman, F.K. Nani, A chemotherapy model for the treatment of cancer with metastasis, Math. Comp. Model., 36 (2002), 773-803.

[16] P. Pisani, F. Bray, D.M. Parkin, Estimates of the world-wide prevalence of cancer for 25 sites in the adult population, Int. J. Cancer, 97 (2001), 72-81.

[17] E.A. Reis, L. Santos, S.T.R. Pinho, Cellular automata model for avascular solid tumor growth under the effect of therapy, Physica A: Statist. Mechanic Appl., 338 (2009), 1303-1314.

[18] D.S. Rodrigues, P.F.A. Mancera, S.T.R. Pinho, A simple mathematical model for vascular tumours under different chemotherapy schedules, Preprint, 1-45, 2011.

[19] H.E. Skipper, F.M. Schaebel-Jr., W.S. Wilcox, Experimental evaluation of potential anticancer agents XIII: on the criteria and kinetics associated with curability of experimental leukemia, Cancer Chemother. Rep., 35, (1964), 1111.

[20] J.S. Spratt, J.S. Meyer, J.A. Spratt, Rates of growth of human neoplasms: part II, J. Surg. Oncol., 61 (1996), 68-73.

[21] G.S. Stamatakos, E.A. Kolokotroni, D.D. Dionysiou, E.C. Georgiadi, C. Desmedt, An advanced discrete state-discrete event multiscale simulation model of the response of a solid tumor to chemotherapy: mimicking a clinical study, J. Theor. Biol., 266 (2010), 124-139.

[22] V.G. Vaidya, F.J. Alexandro-Jr, Evaluation of some mathematical models for tumor growth, Int. J. Bio-Med. Comp., 13 (1982), 19-35.

[23] R. A. Weinberg, "A Biologia do Câncer", Porto Alegre, Tradução Bruna Selbach et al. Artmed, 2008. 